\title{
Prevalence of daytime sleeping in the working population from estimates of nocturnal work shift
}

\author{
Carlos Siordia*1, Athena K Ramos ${ }^{2}$ \\ ${ }^{1}$ Center for Aging and Population Studies, Graduate School of Public Health, University of Pittsburgh, PA, USA \\ ${ }^{2}$ Center for Reducing Health Disparities, College of Public Health, University of Nebraska, NE, USA
}

Received: July 13, 2015

DOI: $10.5430 /$ jer.v1n1p44
Accepted: August 11, 2015

URL: http://dx.doi.org/10.5430/jer.v1n1p44

\begin{abstract}
Background: Sunlight has been linked the circadian rhythms that regulate sleep. Few studies have attempted to provide estimates on the size of the "daytime sleeper" population. Specific aims: Estimate prevalence of daytime sleepers in the labor force population and identify which demographic characteristics are risk factors for daytime sleeping.

Methods: Cross-sectional, community-dwelling, nationally representative, observational study used information on 6,405,063 labor force participants representing 132,682,344 individuals in the contiguous United States. Data from the American Community Survey (ACS), Public Use Microdata Sample (PUMS), 2009-2013 (5-year) file was used to identify daytime sleepers (i.e., those who arrived at work between 7:00 PM and 2:59 AM).

Findings: While nighttime sleepers represented $65.9 \%(n=87,426,814)$ of those in the labor force population, daytime sleepers represent 3.3\% ( $\mathrm{n}=4,344,311)$. Race-ethnic minority status, being disabled, and having low levels of educational attainment were found to be risk factors for daytime sleeping.

Conclusions: Even though relatively small, the objectively large $(\approx 4.3$ million $)$ number of daytime sleepers requires sleep research to invest resources in understanding how health varies in this population relative to those who primarily sleep in the absence of sunlight.
\end{abstract}

Key Words: Demography, Population estimates, Social inequality, Social determinants

\section{INTRODUCTION}

This investigation uses conceptual frameworks and techniques from social demography and epidemiology to estimate the prevalence of daytime sleeping in the communitydwelling population of the United States (US) who participates in the labor force. Under the empirically-informed assumption that sleep habits have the potential to influence population health, the multidisciplinary approach in this analysis aimed to inform public health on the prevalence of and risk for daytime sleeping. This is important because scientific investigations have suggested sleep is influenced by two general physiological systems: sleep/wake homeostasis and the circadian clock. ${ }^{[1]}$ Homeostasis, as a restorative process, drives the balance between sleep and wakefulness. The circadian rhythm contributes to sleep patterns by regulating the timing of periods of sleepiness and wakefulness throughout a 24-hour period. ${ }^{[2]}$ For some people, the circadian rhythm begins to decline a few hours after sunset (e.g., 10:00 PM) and to increase during sunrise (e.g., 7:00 AM). ${ }^{[3]}$

The circadian rhythm has been found to be associated with behavior, physiology, and biochemistry for most living systems. ${ }^{[2]}$ Sunlight exposure is important because the circadian

*Correspondence: Carlos Siordia; Email: cas271 @ pitt.edu; Address: 130 North Bellefield Ave, Pittsburgh, PA 15213, USA. 
rhythm may be influenced by eukaryotic and prokaryotic organisms - which are influenced by light and temperature in the environment. ${ }^{[2]}$ Consequently, it may be that daytime sleepers, because they attempt to sleep during sunlight hours, are at greater risk for adverse health outcomes induced by sleep abnormalities. Sunlight has played a key role in the evolution of life on earth. As a result, most humans synchronize their circadian clock to the "solar day". ${ }^{[4]}$ Sunlight exposure is considered an important environmental cue capable of resetting the circadian rhythm within an individual's endogenous "day" cycle. ${ }^{[5]}$

Since the invention of the artificial lighting that more readily allows being awake without sun-stimuli, the 24-hour light-dark cycle in nature has been altered. Being exposed to "irregular light environments" has been associated with sleep pattern alterations that may lead to mood and cognitive deficits. ${ }^{[4]}$ Deep and prolonged sleep may be more difficult to achieve during sunlight hours when sounds and atmospheric temperature are higher. ${ }^{[6]}$ Working during the night means individuals must sleep during sun-filled hours. A recent review of the literature found sunlight may influence quality of sleep by affecting brain alertness. ${ }^{[7]}$ Thus, working during the night means individuals must sleep during noise-, warm-, and sunlight-filled hours. If sleeping during sun-filled hours is capable of altering the physiological equilibrium of circadian or ultradian rhythms, then estimating the prevalence of and risk for being a "daytime sleeper" is important. It may be that daytime sleepers are at greater risk for adverse health outcomes associated with diseases processes partially induced by sleep abnormalities (e.g., cardiovascular health). The specific aims of this multidisciplinary study were to estimate the prevalence of daytime sleepers and identify risk factors for being a daytime sleeper.

\section{Participants \& methods}

The analysis used information on individuals from the American Community Survey (ACS) Public Use Microdata Sample (PUMS) 2009-2013 (5-year) file. The ACS is a nationally representative survey study conducted by the US Census Bureau. PUMS files are available to anyone with an internet connection and their use does not require IRB approval. Because the ACS is meant to provide estimates of the population, every inhabited housing unit in the US is theoretically given an equal chance of selection in the ACS. The US Census Bureau, the federal agency charged with administering the ACS, does not obtain consent as participation with the survey is framed as being legally mandated of all US residents. The sophisticated sampling, ${ }^{[8]}$ validity, ${ }^{[9]}$ policy value, ${ }^{[10]}$ and high quality of ACS PUMS files have been discussed before. ${ }^{[11]}$ From the 15,450,262 observations in

Published by Sciedu Press the microdata, 8,927,386 (58\%) resided in the contiguous US and reported participating in the labor force. After retaining those with responses on the items identifying their approximate time of sleeping, a total of 6,405,063 (41\%) actual observations were retained for the current analysis. After population weights were applied, these were said to represent 132,682,344 community-dwelling labor force participating individuals within the US mainland who did not work from home during the five-year survey period.

Potential sleep times were identified by creating four work shifts using information from the time of arrival at work composite variable in ACS PUMS file. The time of arrival to work variable was estimated by answers provided to the following two questions: "What time did this person usually leave home to go to work last week?" and "How many minutes did it usually take this person to get from home to work last week?" The US Census Bureau collects information on commuting patterns in order to help inform public transit agencies, metropolitan planning organizations charged with managing traffic problems, police and fire departments needing to estimate population-traffic densities to plan for emergency services. This is the first analysis to use commuting responses to estimate the daytime sleeping population. Novel approaches may provide unique insight.

Three general assumptions were made: most work shifts last about 8 hours; most people wake up about 1 hour before work; most people start sleeping about 3 hours after leaving work; and most people sleep about 7 hours per-day cycle. The assumptions are not guided by empirical evidence; however, we will provide a discussion on how multiple combinations (using slightly different assumptions) yielded the same set of findings and how future research could help establish empirically informed assumptions about average behavioral patterns before and after waking. Daytime sleepers were identified as those who arrived to work from 7:00PM to 2:59AM. Thus, 7:00 PM arrivers are estimated to start attempting sleep at around 6:00 AM (1:00 PM) and 2:59 AM arrivers to start attempting sleep at around 1:59 PM (and rise at 8:59 PM). Mix-evening sleepers are those who are estimated to have arrived to work from 3:00AM to 6:59AM, nighttime sleepers (presumably the group with the most "normal" circadian rhythm) are those who arrived to work from 7:00 AM to 2:59 PM, and mix-morning sleepers those who arrived to work from 3:00 PM to 6:59 PM.

Estimates on the prevalence of non-US-citizens, disability status, ability to speak English not well or at all, marital status, having a high school education and below, and being in-poverty were provided for each of the four work shifts. Poverty status was measured using the Income-to-Poverty 
Ratio (IPR) variable in the data. The IPR captures how total family income compared with the federally established "poverty thresholds" for the family type. For example, in 2013 , the poverty threshold for a family of four was $\$ 23,550$. Race-ethnic groups were also stratified by work shifts using the following groups: Non-Hispanic-White; Non-HispanicBlack; Non-Hispanic-Other; Hispanic of Mexican-origin; and Hispanic of non-Mexican-origin. The coding of all these variables is customary. ${ }^{[8-11]}$ Race-ethnic minority status, disabled status, and educational attainment are included as covariates in our exploratory analysis as these have been frequently found to help explain health disparities.

Although the distribution of the 6,405,063 unweighted counts is provided by work shift to estimate approximate hour range when person attempts to sleep, only population-weighted estimates of prevalence and estimated risk for being a daytime sleeper are provided. Because between-group comparisons were not of interest in the investigation, the statistical significance of the difference in prevalence of daytime sleepers between the different groups was not ascertained in the population-weighted analytic sample of 132,682,344 individuals. Because of the very large sample size, most comparisons would be inferred to be statistically significant at the $<0.05$ level using the frequentist approach to statistics. The
SURVEYLOGISTIC procedure in SAS $\mathbb{R} 9.3$ was used to estimate how different demographic characteristics were associated with the likelihood of being a daytime sleeper versus being a mix-evening, night, or a mix-morning sleeper. Neither a stratum nor cluster variable was used and "PWGTP" was the weight variable in the population-weighted multivariable logistic regression.

\section{RESULTS}

From Table 1, we see daytime sleepers only represent $3.3 \%$ ( $n=4,344,311$ ) of the population in the labor force. The vast majority of individuals are nighttime sleepers $65.9 \%(\mathrm{n}=$ $87,426,814$ ) - the group which is presumed to be at lowest risk for sleep abnormalities induced from sunlight stimuli. Daytime sleepers have the highest prevalence of disability $(6.6 \%)$. They have the lowest proportion $(53.8 \%)$ with a high school diploma or below but the highest prevalence of poverty $(20.3 \%)$. Non-Hispanic-Blacks (19.8\%) are overly represented in the daytime sleeper group. In contrast, nighttime sleepers have the largest proportion with some college or beyond (69.5\%), lowest prevalence of disability (4.9\%), lowest prevalence of limited English language proficiency $(4.1 \%)$, and largest concentration of Non-Hispanic-Whites $(67.6 \%)$.

Table 1. Unweighted and population-weight counts and demographics

\begin{tabular}{|c|c|c|c|c|}
\hline & $\begin{array}{l}\text { Starts Work } \\
7 \text { PM-2:59 AM }\end{array}$ & $\begin{array}{l}\text { Starts Work } \\
\text { 3 AM-6:59 AM }\end{array}$ & $\begin{array}{l}\text { Starts Work } \\
7 \text { AM-2:59 PM }\end{array}$ & $\begin{array}{l}\text { Starts Work } \\
\text { 3 PM-6:59 PM }\end{array}$ \\
\hline & $\begin{array}{l}\text { Day } \\
\text { Sleepers }\end{array}$ & $\begin{array}{l}\text { Mix-Evening } \\
\text { Sleepers }\end{array}$ & $\begin{array}{l}\text { Night } \\
\text { Sleepers }\end{array}$ & $\begin{array}{l}\text { Mix-Morning } \\
\text { Sleepers }\end{array}$ \\
\hline Unweighted & 200,542 & $1,568,122$ & $4,235,305$ & 401,094 \\
\hline Population-weighted & $4,344,311$ & $32,069,170$ & $87,426,814$ & $8,842,049$ \\
\hline Non-US-Citizen & $9.6 \%$ & $9.7 \%$ & $8.5 \%$ & $9.4 \%$ \\
\hline Disabled & $6.6 \%$ & $5.7 \%$ & $4.9 \%$ & $5.4 \%$ \\
\hline Speaks English not well/at all & $5.1 \%$ & $6.1 \%$ & $4.1 \%$ & $5.0 \%$ \\
\hline High school or below & $53.8 \%$ & $54.5 \%$ & $69.5 \%$ & $54.4 \%$ \\
\hline In-poverty & $20.3 \%$ & $13.5 \%$ & $13.6 \%$ & $25.9 \%$ \\
\hline Non-Hispanic-White & $61.0 \%$ & $66.1 \%$ & $67.6 \%$ & $59.9 \%$ \\
\hline Non-Hispanic-Black & $19.8 \%$ & $11.2 \%$ & $10.0 \%$ & $13.0 \%$ \\
\hline Non-Hispanic-Other & $6.6 \%$ & $5.4 \%$ & $7.9 \%$ & $7.6 \%$ \\
\hline Hispanic-Mexican-Origin & $8.9 \%$ & $12.4 \%$ & $8.5 \%$ & $11.6 \%$ \\
\hline Hispanic-non-Mexican & $5.3 \%$ & $5.5 \%$ & $5.8 \%$ & $6.1 \%$ \\
\hline Age $\leq 24$ & $16.5 \%$ & $9.8 \%$ & $11.8 \%$ & $38.0 \%$ \\
\hline Age $25-44$ & $44.6 \%$ & $42.9 \%$ & $44.9 \%$ & $36.7 \%$ \\
\hline Age 45-64 & $35.5 \%$ & $43.5 \%$ & $38.7 \%$ & $22.4 \%$ \\
\hline Age $\geq 65$ & $3.4 \%$ & $3.9 \%$ & $4.5 \%$ & $2.9 \%$ \\
\hline
\end{tabular}


Table 2 shows regression results. The population-weighted multivariable regression modelled the likelihood of being a daytime sleeper versus all other conditions (i.e., mixevening, night, and mix-morning sleepers). In terms of race and ethnicity when compared to the majority group in the US (i.e., Non-Hispanic-Whites), Non-Hispanic-Blacks had higher odds $(\mathrm{OR}=1.93 ; \alpha<0.001)$ of being a daytime sleepers. In similar manner, Non-Hispanic-Others $(\mathrm{OR}=1.26 ; \alpha$ $<0.001)$, Hispanic-non-Mexicans $(\mathrm{OR}=1.12 ; \alpha<0.001)$, and Hispanic-Mexican-Origin ( $\mathrm{OR}=1.06 ; \alpha<0.001)$ had higher odds of being a daytime sleepers than Non-HispanicWhites. These findings suggest race-ethnic minority group members may be at greater risk for experiencing abnormal sleep patterns relative to Non-Hispanic-Whites.

Table 2. Population-weighted multivariable logistic regression results

\begin{tabular}{lllll}
\hline & $\mathbf{O R}^{\Delta}$ & $\mathbf{L C L}^{\#}$ & $\mathbf{U C L}^{\dagger}$ & \\
\hline Non-Hispanic-White & 1.00 & Ref & Ref & \\
Non-Hispanic-Black & 1.93 & 1.90 & 1.96 & $* * *$ \\
Non-Hispanic-Other & 1.26 & 1.24 & 1.29 & $* * *$ \\
Hispanic-Mexican-Origin & 1.06 & 1.03 & 1.08 & $* * *$ \\
Hispanic-non-Mexican & 1.12 & 1.09 & 1.15 & $* * *$ \\
Female & 0.70 & 0.69 & 0.71 & $* * *$ \\
Married & 0.77 & 0.76 & 0.78 & $* * *$ \\
Non-US-Citizen & 0.93 & 0.91 & 0.96 & $* * *$ \\
Disabled & 1.25 & 1.23 & 1.28 & $* * *$ \\
High school or below & 1.49 & 1.47 & 1.51 & $* * *$ \\
English not well/at all & 0.96 & 0.93 & 0.99 & $*$ \\
Age & 0.99 & 1.00 & 1.01 & $* * *$ \\
\hline
\end{tabular}

${ }^{*} p \leq .05 ; * * * p \leq .001$

${ }^{\Delta}$ Odds ratio $=\mathrm{e}^{\beta} ;{ }^{\#} 95 \%$ Wald lower confidence limit; ${ }^{\dagger}$ 95\% Wald upper confidence limit

\section{Discussion}

The interdisciplinary investigation used conceptual frameworks and techniques from social demography and epidemiology to estimate the prevalence of daytime sleeping in the community-dwelling population participating in the labor force of the US. Results indicate daytime sleeping is prevalent in about 3.3\% $(\mathrm{n} \approx 4,344,311)$ of individuals in the labor force within the US mainland. Those of race-ethnic minority status, disabled status, or with low educational levels were found to be at risk for being daytime sleeping. These findings can inform the sleep health literature by showing that even though only $3.3 \%$ of the labor force population in the US ( $\mathrm{n} \approx 132,682,344)$ was made-up of daytime sleepers, variance in risk for being a daytime sleeper was explained by demographic factors (e.g., race-ethnicity). Research should continue to identify social determinants of sleep abnormalities in order to better inform clinical practitioners and policy

Published by Sciedu Press makers on the characteristics of vulnerable populations.

There are limitations with this study. For example, empirical methods should be used to determine if the assumptions on how people work and sleep (most work 8 hours, wake up about 1 hour before work, sleep 3 hours after leaving work, and sleep 7 hours) are representative of the labor force population in the US. Although not shown here a complex algorithm was created to produce all possible combinations $(2 \times 2 \times 2 \times 2=16)$ of these assumptions by +1 hour and the results remained the same. The study is also limited in that it only measures potential daytime sleeping in the last week of work. Future studies should investigate how labor force participants enter, remain, and/or exit night-time working over longer periods of time. ${ }^{[12,13]}$ In addition, disability is measured as a non-specific dimension - i.e., without regard to type and severity. Future work should explore is disaggregating disability by type and severity to help inform this line of research.

The study is also limited in that it did not account for the occupation $^{[14]}$ of the daytime sleeper - although educational attainment was included. The absence of occupation in the explanatory variables is a notable issue because, together with working part-time or full-time, certain occupations may have an over-concentration of persons from socioeconomically disadvantaged backgrounds. Should occupation turn out to be the driving force differentiating individuals, it may suggest the real predictor is occupation. Future work should explore using available occupational categorization variables in the ACS PUMS file. In addition, research should continue to explore if there is a causal relationship between daytime sleeping and adverse health induced by sleep-related abnormalities. In addition, attributes of the sleeping environment - including the home structure and surrounding neighborhood-are likely to play a role in quantity and quality of sleep. No attempts were made to include environmental measures. Although ACS data can only be geographically referenced to the Public Use Microdata Area (PUMA) geography, ${ }^{[15]}$ PUMAs are for the most part very large and complex polygons. ${ }^{[16]}$ The geographical attributes of PUMAs would render measurements of "environmental exposures" difficult to interpret in the likely presence of spatial mismatch.

\section{Conclusion}

Notwithstanding limitations, the investigation uses more than 6.4 million observations to generalize findings of prevalence and risk for daytime sleeping to 132.7 million people. The investigation is novel in that it uses the largest survey study data available in the US to infer the size of the population at risk for potential sleep abnormalities including those attempting to complete most of their sleep during sunlight hours. 
The study is creative because it uses information on work commuting patterns to estimate the number of people who work during the night and sleep during the day. The findings support previous work highlighting that socioeconomically vulnerable populations (e.g., Non-Hispanic-Blacks) may be at greater risk for sleep abnormalities, ${ }^{[17-19]}$ a sub-group of individuals known to have greater environmental stressors throughout the lifecourse. ${ }^{[20-22]}$ Sleep research should continue to study the relatively small $(\approx 3.3 \%)$, but objectively large ( $\approx 4.3$ million), understudied population of individuals who attempt to complete all their sleep during sunlight hours. This group of sleepers may provide unique insight on how sunlight, daytime noise, and atmospheric temperature play a role in the etiology of disease over the lifecourse.

\section{REFERENCES}

[1] Lewis S. Circadian rhythms: Remembering night and day. Nature Reviews Neuroscience. 2014; 16(3). http://dx.doi.org/10.10 $38 / \mathrm{nrn} 3885$

[2] Wetterberg L. Light and biological rhythms in man. Elsevier; 2014.

[3] May CP. It's About Time: Circadian Rhythms, Memory, and Aging. Page 199 in Human Learning and Memory: Advances in Theory and Applications: The 4th Tsukuba International Conference on Memory. Psychology Press; 2014.

[4] LeGates TA, Fernandez DC, Hattar S. Light as a central modulator of circadian rhythms, sleep and affect. Nature Reviews Neuroscience. 2014; 15; 443-54. PMid:24917305. http://dx.doi.org/10.10 $38 / \mathrm{nrn} 3743$

[5] Perez-Santángelo S, Mancini E, Francey LJ, et al. Role for LSM genes in the regulation of circadian rhythms. Proceedings of the National Academy of Sciences. 2014; 111(42): 15166-71.

[6] van Alphen B, Allada R. Knock, Knock to Reset the Clock: Mechanosensation and Circadian Rhythms. Cell Metabolism. 2014; 19(5): 739-40. PMid:24807220. http://dx.doi.org/10.1016/j .cmet.2014.04.011

[7] Gaggioni G, Maquet P, Schmidt C, et al. Neuroimaging, cognition, light and circadian rhythms. Frontiers in Systems Neuroscience. 2014; 8.

[8] Siordia C. Demographic, Economic, Household, and Health Profile of Grandparents Responsible for Grandchildren. Journal of Child \& Family Studies. 2014: 1-7.

[9] Siordia C. Married Once, Twice, and Three or more Times: Data from the American Community Survey. Journal of Divorce \& Remarriage. 2014; 55: 206-15.

[10] Siordia C, Young RL. Methodological Note: Allocation of Disability Items in the American Community Survey. Disability \& Health Journal. 2013; 6(2): 149-53.

[11] Siordia C. Sex-Specific Disability Prevalence in Immigrants from China, India, and Mexico and their US-Born Counterparts. International Journal of Health Sciences \& Research. 2015; 5(4): 267-79.
[12] Stamatakis KA, Kaplan GA, Roberts RE. Short sleep duration across income, education, and race/ethnic groups: population prevalence and growing disparities during 34 years of follow-up. Annals of Epidemiology. 2007; 17(12): 948-55. PMid:17855122. http://dx.doi.org/10.1016/j . annepidem.2007.07.096

[13] Hale L, Do DP. Racial differences in self-reports of sleep duration in a population-based study. Sleep. 2007; 30(9): 1096. PMid:17910381.

[14] Jackson CL, Redline S, Kawachi I, et al. Racial disparities in short sleep duration by occupation and industry. American Journal of Epidemiology. 2013; 178(9): 1442-51.

[15] Siordia C, Wunneburger DF. On the Quantity, Degree, and Location of Public Use Microdata Area (PUMA) Fragmentation. Human Geographies. 2013; 7(2): 5-13. http://dx.doi.org/10.5719/hge 0.2013 .72 .5

[16] Siordia C, Fox A. Public Use Microdata Area Fragmentation: Research and Policy Implications of Polygon Discontiguity. Spatial Demography. 2013; 1(1): 42-56.

[17] Grandner MA, Petrov MER, Rattanaumpawan P, et al. Sleep symptoms, race/ethnicity, and socioeconomic position. Journal of clinical sleep medicine. JCSM: official publication of the American Academy of Sleep Medicine. 2013; 9(9): 897.

[18] Hicken MT, Lee H, Ailshire J, et al. "Every shut eye, ain't sleep": The role of racism-related vigilance in racial/ethnic disparities in sleep difficulty. Race and Social Problems. 2013; 5(2): 100-12.

[19] Williams NJ, Grandner MA, Wallace DM, et al. Social and behavioral predictors of insufficient sleep among African Americans and Caucasians. Sleep Medicine. 2015. http://dx.doi.org/10.10 16/j.sleep. 2015.02.533

[20] Kingsbury JH, Buxton OM, Emmons KM, et al. Sleep and its relationship to racial and ethnic disparities in cardiovascular disease. Current Cardiovascular Risk Reports. 2013; 7(5): 387-94.

[21] Givens ML, Malecki KC, Peppard PE, et al. Shiftwork, sleep habits, and metabolic disparities: results from the Survey of the Health of Wisconsin. Sleep Health. 2015; 1(2): 15-20.

[22] Laposky AD, Van Cauter E, Diez-Roux AV. Reducing health disparities: the role of sleep deficiency and sleep disorders. Sleep Medicine. 2015. http://dx.doi.org/10.1016/j.sleep. 2015.01.007 